\title{
The basics of endoscopic retrograde cholangiopancreatography: Getting there and getting in
}

\author{
DAVID S ZIMMON, MD, FACP
}

DS ZIMMON. The basics of endoscopic retrograde cholangiopancreatography: Getting there and getting in. Can J Gastroenterol 1993;7(6):498-502. An overview of endoscopic retrograde cholangiopancreatography methods and the associated pitfalls is presented in narrative form, with accompanying protocols for patient and equipment preparation and lists of important technical sequences and clinical scenarios. A step-by-step reconstruction of the manoeuvres required for duct opacification, selective duct cannulation and the subsequent therapeutic techniques is provided. Pitfalls are identified as failure scenarios with suggested methods for avoidance or countermeasures. This paper aims to identify and avoid correctable oversights and technical errors that lead to failed procedures or complications by providing checklists and problem scenarios with their solutions.

Key Words: Complications, Endoscopic retrograde cholangiopancreatography (ERCP), ERCP technique, Pitfalls

\section{Éléments de base de la cholangiopancréatographie endoscopique rétrograde}

RÉSUMÉ: Un tour d'horizon des méthodes de cholangiopancréatographie endoscopique rétrograde et des écueils qui y sont associés sont présentés sous forme narrative avec les protocoles nécessaires pour la préparation des patients et du matériel, des marches à suivre et des scénarios cliniques. Une revue des différentes étapes à franchir pour obtenir l'opacification du canal, pour le canuler et des techniques thérapeutiques subséquentes est présentée. Les pièges sont identifiés sous forme de scénarios d'échec, de même que des méthodes et des mesures pour prévenir ou contrer ces problèmes. Au moyen de listes de vérification et de scénarios pratiques avec leurs solutions, cet article vise à identifier et à permettre d'éviter les problèmes et les erreurs techniques qui entraînent l'échec de l'intervention ou des complications.

Department of Medicine and Radiology, St Vincent's Hospital and Medical Center, New York, New York, USA

Correspondence and reprints: Dr DS Zimmon, Department of Medicine and Radiology, St Vincent's Hospital and Medical Center, 36-7th Avenue, Suite 516, New York, NY 10011, USA
HE ART OF ENDOSCOPIC RETRO.
grade cholangiopancreatography (ERCP) is a complex meld of physiology, endoscopy, microsurgical manipulation and radiography with fluoroscopy. My experience with ERCP dates from 1971. The first 1000 cases were reported in 1976 (1). My experience over the past 22 years includes approximately 10,000 procedures that form the basis of the impressions and conclusions presented here.

Learning ERCP now is more difficult than in the past because of the absence of a large volume of diagnostic procedures. When ERCP was introduced in 1968, neither ultrasound nor computed tomography were available. Consequently, the current role played by these useful diagnostic procedures was taken by diagnostic ERCP. Furthermore, current therapeutic procedures were not practiced so that therapy often was surgical. Endoscopic sphincterotomy was first performed in Europe in 1974 and in the United States in 1975. Thus goals were limited to diagnostic ERCP. This narrow focus allowed time and opportunity for the trainee to learn and even fail without great penalty to the patient since the preceptor could almost always salvage 


\section{TABLE 1}

\section{Protocol for successful endoscopic retrograde cholangiopancreatography}

\begin{tabular}{|c|c|c|}
\hline Patient preparation & Tilt table & Identifying papilla \\
\hline Mental preparation: informed, confident & Developing & endoscopy: aperistalsis, distension \\
\hline Cardiovascular: respiratory stability* & Delay & fluoroscopy: junction 2 and 3 duodenum \\
\hline Medication & Recovery & variants: high and low \\
\hline vital (beta-block) & Site & Cannulating \\
\hline optional (acetylsalicylic acid) & Monitoring & J position \\
\hline Allergy: contrast, prophylactic therapy & Laboratory & patient relaxation: gut, papilla \\
\hline Bowel preparation & Follow-up & close adjustments \\
\hline colon & Diagnostic, therapeutic, sphincterotomy & catheter position \\
\hline stomach & Endoscope(s) & parallel to bile duct (11:00 to 12:00) \\
\hline Smoking & Dx $2.8 \mathrm{~mm}$ channel & parallel to pancreatic duct ( $5: 00$ to $6: 00$ ) \\
\hline Anesthesia & $\mathrm{R} \times 4.2 \mathrm{~mm}$ channel & catheter injection positions \\
\hline oral topical & Video versus optical & blocking \\
\hline neuroleptic, endotracheal (rarely) & Backup endoscope & simultaneous biliary and pancreatic \\
\hline none & Elevator function & pancreatic \\
\hline Monitoring & Disinfection - cleanliness versus sterility & biliary \\
\hline pulse oximeter & Cautery: Polypectomy versus sphincterotomy & alternate duct \\
\hline electrocardiogram & Endoscopic devices & alternate catheter \\
\hline Antibiotics & Procedure & metal tip \\
\hline percutaneous alternative & Patient position: tilt, passage, cannulation & long taper tip \\
\hline prophylaxis $^{\dagger}$ & Drugs & selective duct cannulation \\
\hline Endoscopist & anticholinergic & standard catheter \\
\hline Time and patience & glycopyrrolate 0.2 & endoscopic line \\
\hline X-ray gown and radiation monitor & buscopan & fluoroscopic line \\
\hline Shields & glucagon & 35K soft tip wire guide \\
\hline Associates and assistants & secretin & slip wire \\
\hline Radiologist & sedatives & stent \\
\hline$X$-ray technician & reversal medication & alternate duct stenting, sphincterotomy \\
\hline Gastrointestinal assistant & Endoscopy & infundibulotomy \\
\hline Anesthesiologist & esophagus & percutaneous transhepatic wire guide \\
\hline Circulating assistant & gastric aspiration & precut sphincterotomy \\
\hline X-ray equipment & passing pylorus & abandon and repeat \\
\hline Fluoroscopy quality and magnification & straightening & refer \\
\hline Radiographic spot and overhead & fluoroscopy assistance & surgery \\
\hline
\end{tabular}

"Workup in advance: ${ }^{\dagger}$ Cardiovascular, pancreatic, biliary

the brief, relatively simple diagnostic cholangiogram or pancreatogram that was needed.

Now, in most cases (approximately $80 \%$ ), the diagnostic ERCP is followed immediately by a therapeutic procedure. Consequently, the risk of complications and failure is considerably increased compared with when the only goal was diagnostic ERCP. Many complications of diagnostic ERCP, such as cholangitis or pancreatitis, are obviated or limited by the therapeutic procedure that should immediately follow the diagnostic cholangiogram and or pancreatogram (2). For example, the demonstration of a common duct stone demands immédiate biliary decompression by either endoscopic sphinctero- tomy and stone removal, or insertion of a stent or drain if cholangitis and/or pancreatitis are to be avoided. Furthermore, the risks of both morbidity and mortality in the alternative percutaneous transhepatic or surgical operative procedures are substantially greater than therapy by endoscopy. This places a premium on success, both diagnostic and therapeutic.

From a technical standpoint, only a short period of trial and error often is available in ERCP before aperistalsis is lost, or edema of the minute orifices of the biliary or pancreatic ducts occurs, and even an expert can not salvage the situation. Therefore attention to minute details of technique is essential for technical success and to avoid compli- cations. Several ERCP information sources describe or illustrate what to do, but there is little information on how to do it. Of necessity, my views in these matters are personal and, undoubtedly, idiosyncratic. I often hear or read statements by individuals who are experienced in this field that I strongly disagree with. How is one to know if their success or complication rate is slightly better or worse than another? Undoubtedly there is more than one path to success.

Every procedure is modified by the anatomical and clinical situation of the individual patient, as well as the patient's reaction to the intervention. Few of these phenomena can be foreseen in advance and, therefore, consid- 


\section{TABLE 2}

\section{Patient position}

\begin{tabular}{ll}
\hline Endoscopy & Left lateral decubitus \\
Straighten & Prone, head up \\
Locate papilla & Prone, head up \\
Cannulate & Prone, head up \\
Final & Supine, horizontal; \\
\multicolumn{1}{c}{ overhead } & right lateral \\
x-ray & $\begin{array}{l}\text { decubitus; left } \\
\text { lateral decubitus }\end{array}$ \\
\hline
\end{tabular}

erable expertise is required to react appropriately. Recognizing a situation that must be corrected to succeed and matching that situation with the appropriate corrective response is the art of ERCP that can only be gained with experience.

The following gives a description of my general methods for performing these procedures but, of necessity, can not be all inclusive or definitive. Nevertheless, 1 hope that by reading this, those inexperienced in the field will gain at least a checklist to avoid some of the pitfalls, improve their success rate and reduce their incidence of complications. The protocol attempts to identify, in outline form, the considerations that I consider vital for successful procedures and avoiding complications (Table 1).

\section{PATIENT PREPARATION} Cardiovascular and respiratory stability: In preparation for ERCP, it is important that the patient has confidence in the operator and appreciates the value of the procedure compared with the risk and discomfort. I am often surprised to find patients who present on referral for ERCP who still believe it to be a surgical procedure requiring an incision and general anesthesia. Many patients are considerably reassured by the fact that they will not be intubated and that, almost always, mild analgesia and cooperative somnolence produced by a narcotic and a benzodiazepine are all that is required. We rarely use endotracheal anesthesia unless there is a strong indication, such as respiratory impairment, or the occasional patient in whom the margin between adequate sedation with cooperation and respiratory dysfunction is narrow. The vast major-

TABLE 3

\section{Opacification sequence}

1. Find papilla

2. Straighten endoscope to $60 \mathrm{~cm}$

3. Patient: prone, still

4. Aperistalsis

5. Papilla relaxation

6. Endoscope position: close, en face

7. Catheter position: short

8. Injection
a) blocking
b) common channel
c) pancreatic
d) billary

ity of our anesthesia is given by a nurse gastroenterology assistant without difficulty. Particularly for therapeutic endoscopic procedures, I believe that continuous oximetry and electrocardiographic monitoring are essential, not just to prevent complications of anesthesia (which I believe it sometimes does), but to allow the adequate administration of the appropriate drugs with easy physiological monitoring in a darkened room where attention is diverted from the patient by the complex procedure.

One of the most important factors in preparing a patient for ERCP is the avoidance of smoking, which produces considerable spasm in the gastrointestinal tract and at the sphincter (as well as a respiratory problem). We generally use an anticholinergic such as glycopyrolate since butyl scopolamine is not available in the United States. We supplement with glucagon if necessary, and when the patient has an excessive cardiac rate. In aged or high risk patients, we use glucagon exclusively. Tachycardia may be controlled by short acting beta-block.

It is essential that the patient have an empty upper gastrointestinal tract to avoid aspiration. If the gastrointestinal tract can not be emptied adequately by endoscopic suction, we may abandon the procedure because of the risk of aspiration.

Gastrointestinal tract preparation: It is essential that the patient be fasting. A bowel prep is important because it reduces the volume of intestinal contents, avoids the unpleasant experience of the patient expelling feces during the procedure and makes room for the inevitable air that is insufflated into the gut during the performance of the procedure.

We use an oral local anesthetic lozenge in preference to any of the potentially more toxic spray or gargle anesthetics. In particular, we wish to obtund the gag reflex while avoiding anesthesia of the larynx that occurs with gargles and spray anesthetics. This preserves the patient's cough reflex and prevents aspiration on the occasional reflux of fluid into the hypopharynx. Pharyngeal anesthesia also decreases the amount of intravenous sedation required to pass the endoscope and allows the patient to take liquids soon after the procedure.

Premedication: As previously stated, an informed patient is more relaxed and comfortable than an uninformed one. However, some patients are extremely anxious despite their desire to have the procedure and their appreciation of its necessity. I believe premedication is important. Narcotics given immediately prior to the procedure tend to produce spasm of the papilla of Vater and upper gastrointestinal tract, making the procedure more difficult. We, therefore, administer meperidine $50 \mathrm{mg}$ or more as necessary with a benzodiazepine according to the patient's weight at least $30 \mathrm{mins}$ before the procedure whenever possible.

Patients who travel for ambulatory procedures present a special problem. If they can be seen in advance to obtain informed consent or attend with a family member who can be responsible for them, it is sometimes advantageous to administer a mild oral sedative such as 2 or $5 \mathrm{mg}$ diazepam when the patient leaves home. It is essentially that the patient does not smoke for at least $12 \mathrm{~h}$ prior to the procedure.

During the procedure, additional doses of meperidine and diazepam or similar agents appear to produce atropine-like action that minimizes the need for other anticholinergics or glucagon. This pharmacological synergism yields smooth induction, amnesia, relaxation of the gut and a still patient so that complex therapeutic procedures may be performed rapidly without interruption for sedation or to await aperistalsis. 
Antibiotic prophylaxis: Prophylactic antibiotic therapy clearly is effective in invasive surgical procedures. I consider this fact adequate to support a similar use in therapeutic ERCP. Furthermore, all our patients who accept are prepared for a diagnostic or therapeutic percutaneous procedure as required and therefore should have prophylaxis against the common associated complications of fever or sepsis.

Endoscopes: Large channel endoscopes are essential for some therapeutic procedures but I believe that smaller diameter instruments more easily pass the hypopharynx (particularly in older patients), may occasionally pass a stenosis that cannot be passed with a larger diameter endoscope and are more facile in a narrow duodenum. I still find circumstances where an optical endoscope serves me well and draw comfort from the simplicity of an optical endoscope with a video camera (and a backup light source in case of electronic failure).

Beyond disinfection is the importance of removing all glutaraldehyde that might be carried into the duct systems. There should be no odour of disinfectant from endoscope, wash bottle or devices. Reusable devices should be dry sterilized with ethylene oxide or steam. Particulate matter that is potentially infectious may collect within the instrument, particularly in heavily used endoscopes when the plastic liner is damaged. Before use, the elevator and valves should be inspected and lubricated as necessary. Adherent particulate matter or frozen controls indicate a need for reconsidering disinfection methods or instrument repair. Elevator function is a major technical and maintenance problem. The power of elevation cannot be easily estimated in vitro. A weak elevator makes cannulation difficult and should be noted during a procedure. Backup endoscopes are essential to guard against all types of electronic or mechanical failure.

Cautery: Sphincterotomy requires $75 \mathrm{~W}$ compared with 15 to $20 \mathrm{~W}$ in polypectomy. Consequently, a cautery unit that is satisfactory for the latter may produce too much coagulation with slow cutting during sphincterotomy. The author initiates a papillotomy (incision of the
TABLE 4

\begin{tabular}{lll}
\multicolumn{3}{l}{ Alignment-endoscope and catheter } \\
\hline Position & Biliary & Pancreatic \\
\hline Catheter & $11: 00$ & 5:00 \\
Endoscope & Below, up & Level, down \\
Orifice & Proximal & Distal \\
\hline
\end{tabular}

TABLE 5

\section{Failure scenarios}

Patient anxiety

Cardiovascular or respiratory instability

Smoking - peristalsis

Pharmacological failure-pancreatitis, cirrhosis

Local anatomy - pancreatic cancer

Fluoroscopy limiting - patient, equipment

Endoscope limiting - backlash

Technical error-submucosal contrast

inferior and submucosal sphincters) with sharp cutting current to avoid injury to the adjacent pancreatic duct (3). If a sphincterotomy is necessary (incision of the proximal sphincter), I change to a blended current to produce more coagulation as the upper portion of the intraduodenal bile duct (that may contain branches of the retroduodenal artery) is approached.

Sphincterotomes: A braided wire sphincterotome was developed in preference to the early single wire device to improve coagulation when incising the proximal intraduodenal bile duct. Distal sphincterotomy (papillotomy) (4), particularly when the intraduodenal bile duct is small, risks pancreatitis as a complication, probably due to instrument trauma or coagulation of the pancreatic duct from incision of the adjacent bile duct. This may be avoided by using cutting current without coagulation. Often only a limited incision 'papillotomy' is required to remove a small stone or separate the biliary and pancreatic ducts in gallstone pancreatitis. The greater hazards of longer, more proximal incision can be avoided.

The 'sphincterotomy' incises the thicker, more muscular proximal sphincter unless an infundibulum is present. The small muscular arteries or, in some cases, a retroduodenal artery in the proximal intraduodenal bile duct may require greater cutting power and
TABLE 6

\section{Failure scenarios}

Inadequate anesthesia

retching

air and fluid regurgitation

aspiration

increased anesthesia

excess respiratory motion

duodenal movement

papilla trauma and spasm

Hiatus hernia - reflux

excess gastric fluid (gastric juice.

bile, contrast)

prone position

regurgitation

aspiration

Endoscopic position

long (looped) endoscope

distance from papilla

limited controls

poor approach angle

papilla trauma

gastric distension

coagulation to prevent bleeding. The braided wire - as in colonic polypectomy - allows this by simply changing to a mixed coagulating and cutting current. An alternative would be to select papillotome type according to the local anatomy and desired result. In either case, the concept of 'papillotomy' as distinct in technique, results and risks from 'sphincterotomy' is important.

\section{PROCEDURE}

Patient position: The patient is positioned head up to avoid aspiration, allow insufflated air to escape via the stomach and mouth, and pass contrast into small intestine where it will not overlie the pancreas or bile duct (Table 2 ). The prone position moves the biliary tree and distal pancreas off the spine for fluoroscopic visualization.

Endoscopy: ERCP should start with a diagnostic endoscopy and removal of all secretions from the esophagus and stomach. If necessary, a forward viewing endoscopy may initiate the examination. The standard lateral view duodenoscope usually is adequate to examine all but the upper one-third of the esophagus. In my opinion, visualization of the cardia, lesser curvature and base of the duodenal bulb is superior with the 
lateral view if the light source is adequate.

After passing the pylorus, the endoscope should be straightened and the patient turned into the full prone position; confirmed by fluoroscopy, this assures that the $\mathrm{x}$-ray unit is positioned and prepared. The papillae should be easily identified in most patients by the visible mucosal rosette. Duodenal and sphincter relaxation are then confirmed and, if not adequate, the appropriate drug is administered.

Cannulating: With the patient prone, a combination of adjustments with the up-down $(\mathrm{J})$ control and the lateral control will bring the endoscope close to the papillae in the on face position. These manoeuvres allow a short length of catheter to be manipulated with control of angle and depth of insertion. The opacification sequence requires fluoroscopic control to monitor both the cannulating position and contrast injection (Table 3). The easiest method of opacification is to block the papillary orifice or enter the lumen just enough to prevent contrast leak into the duodenum and simultaneously inject both ducts. Care is required not to overinject the pancreatic duct while attention is diverted to the filling of the larger volume biliary tree. Overfilling of the pancreas may provoke pancreatitis.

Unfortunately, this easy method often fails and only one duct is opacified. Gaining visualization of the other duct and selective duct cannulation for therapeutic procedures requires precise control of endoscope and catheter position to align the catheter to the duct of interest (Table 4). In general, the bile duct passes vertically along the duodenal wall and the pancreatic duct extends medially towards the spine, but within the sphincter the ducts may be parallel. This requires precise angulation of the catheter guided by endoscope positioning to cannulate the desired duct.
Selective duct cannulation: The most difficult judgement in selective duct cannulation is identification of the problem when initial attempts fail. If disease obstructs a duct as indicated by prior imaging studies, one may rapidly advance to the indicated, relatively hazardous but effective sphincterotomy or percutaneous techniques. When the diagnosis is not clear, the low risk endoscopic methods should be pushed to their limit and usually will succeed. This is an area where experience and anatomical or clinical clues are important. I avoid precuts and unnecessary trauma when pancreatitis, sphincter dysfunction (particularly with normal laboratory data), a small papilla or other risk factors, such as age or concomitant disease, are present. The dangers and pain of pancreatitis are, in my view, limited by insertion of a 5 French pigtail-flap pancreatic stent to facilitate a 'precut' sphincterotomy and prevent postsphincterotomy pancreatitis. The duodenal pigtail obviates the disaster of losing the stent into the pancreatic duct. A short stent of 2 to $4 \mathrm{~cm}$, according to the ductal anatomy, allows stent introduction without passing the genu with wire guide or stent and minimizes trauma to a small normal duct.

This said, the best policy still is to study the anatomy and align catheter with duct to first opacify and then advance catheter or a soft tip teflon coated guidewire into the duct. In my experience, slip wires work well in the proximal duct system but require selective duct cannulation before they can be applied. The taper tip catheter with a 0.018 " wire simultaneously opacifies and dilates. It is most useful when the duct is not opacified, otherwise I favour passing a soft tip wire guide into the opacified duct with careful attention to the endoscopic and fluoroscopic line of introduction.

\section{FAILURE SCENARIOS}

Sequences of events that eventuate in failure come to be recognized by experienced operators (Table 5). If one has the sequence in mind, it may be possible to identify it early and correct the cause. With the sequence aborted the procedure may succeed. Table 5 lists a series of problems that are often encountered. These problems must be recognized and corrected to succeed. Failure scenarios (Table 6) are listed sequences of events arising from specific causes, eg, inadequate anesthesia, hiatus hernia and failure to straighten the endoscope.

\section{CONCLUSION}

It is difficult to reduce to words a complex series of actions, observations and hand-eye coordinated acts. Even with a perfect understanding of what is desirable, it often is difficult to be successful at the task. The best form of learning is preceptorship. The Wellesley Hospital Course of live videoendoscopy provides a valuable compromise to days or weeks of overthe-shoulder instruction. I hope this annotated outline adds to the teaching value of the entire effort.

\section{REFERENCES}

1. Kessler RE, Falkenstein DB, Clemett $A R$, Zimmon DS. Indications, clinical value and complications of endoscopic retrograde cholangiopancreatography: Analysis of 1089 consecutive cases. Surg Gynecol Obstet 1976;142:865-71.

2. Zimmon DS, Falkenstein DB, Riccobono C, Aaron B. Complications of endoscopic retrograde cholangiopancreatography (ERCP): Analysis of 300 consecutive cases. Gastroenterology 1975;69:303-9.

3. Zimmon DS, Falkenstein DB, Kessler RE. Endoscopic papillotomy for choledocholithiasis. N Engl J Med 1975;293:1181-2.

4. Zimmon DS. Endoscopic management of biliary calculi. Hosp Prac 1978;13:103-14. 


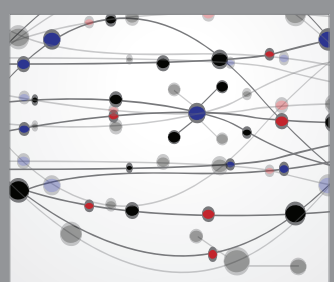

The Scientific World Journal
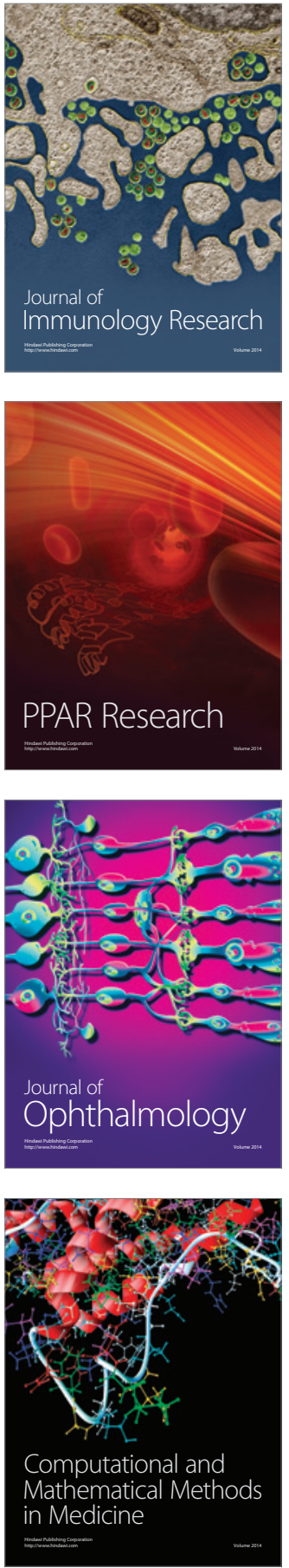

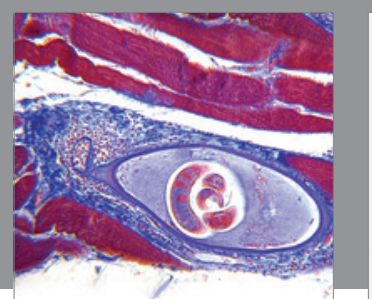

Gastroenterology Research and Practice

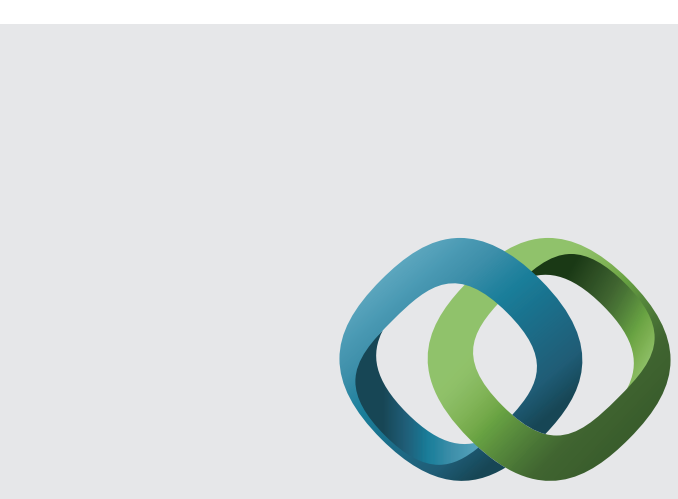

\section{Hindawi}

Submit your manuscripts at

http://www.hindawi.com
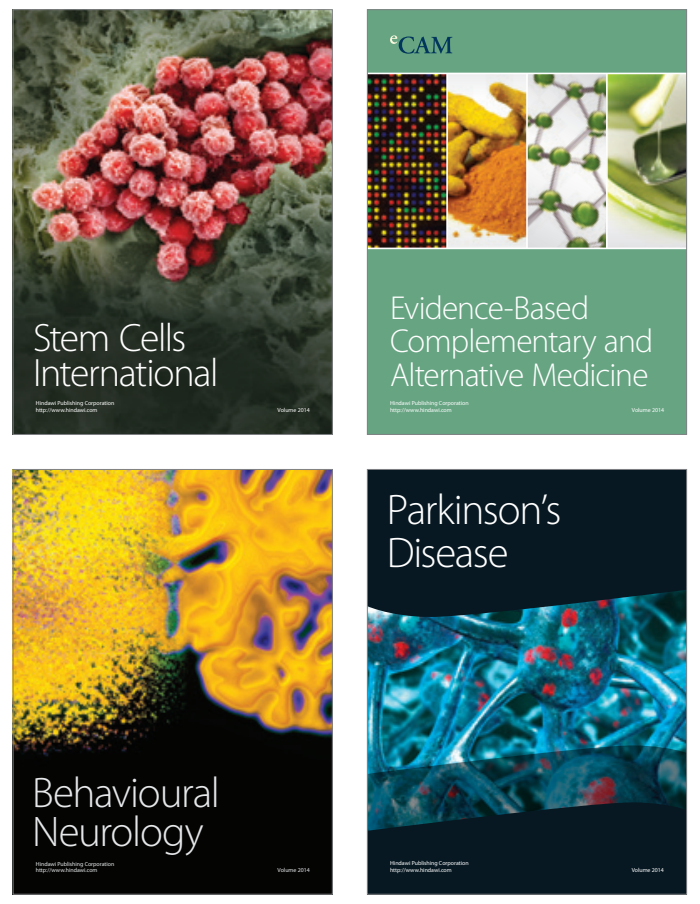
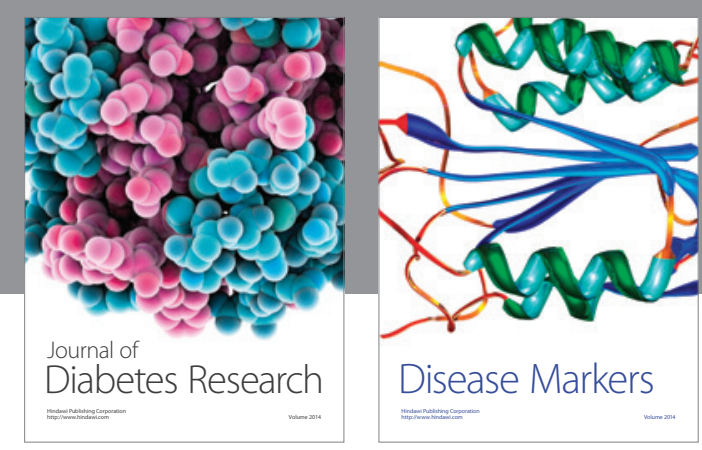

Disease Markers
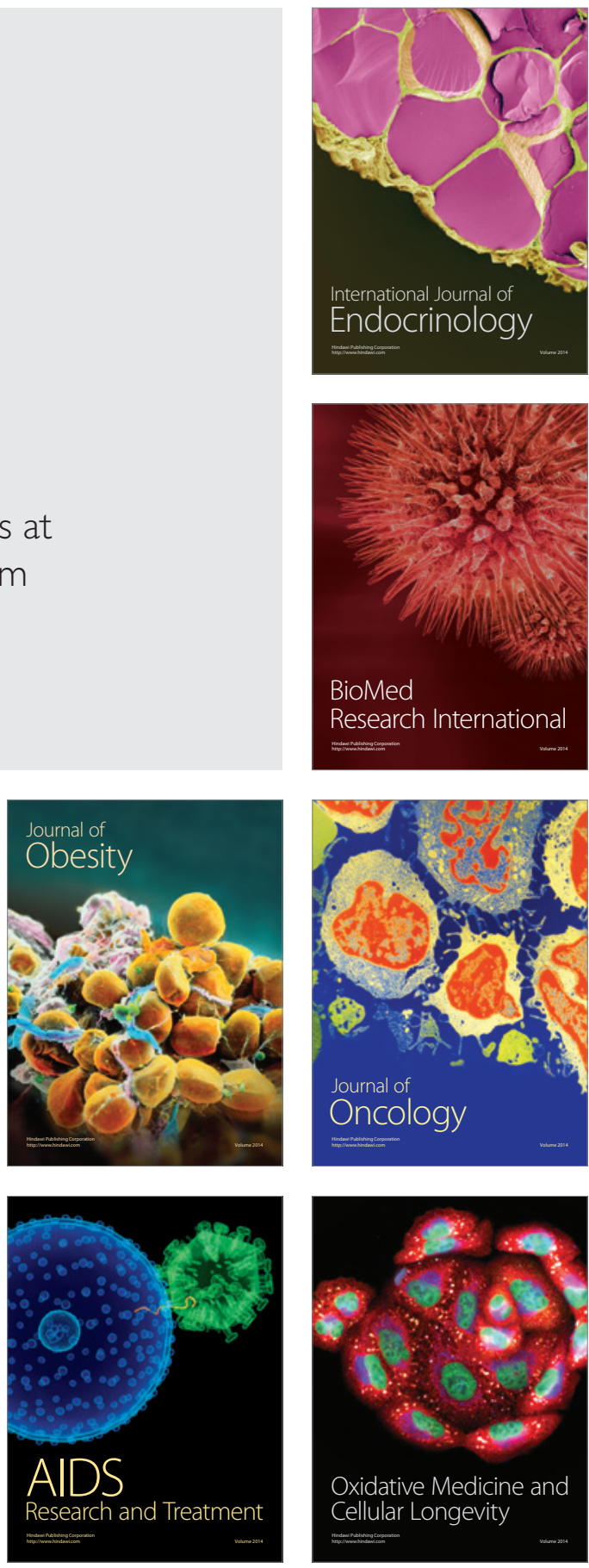